\title{
Discrete Lion Swarm Optimization Algorithm for Face Recognition
}

\author{
Shuang You \\ University of Science and Technology of China \\ yousustc@gmail.com
}

\begin{abstract}
In various applications, face recognition plays an important role such as identification of a person, biometrics via their Closed-Circuit Television (CCTV) cameras, identity cards and etc. Besides, various biometrics like palm print, fingerprint, iris, etc plays a significant role. Therefore, in this research, a face recognition technique is developed for the classification phase as well as feature extraction. Moreover, Discrete Lion Swarm Optimization Algorithm (DLSA) is developed Deep Belief Network (DBN) for face recognition. At first, in the database, the images experience feature extraction here the feature like m-Co-HOG, Kernel based Scale Invariant Feature Transform (K-SIFT), besides with Active Appearance Models (AAM) features which are extracted from the image. Subsequently, developed DLSA-DBN is used for the classification. The simulation of the developed technique is performed by exploiting the CVL database. In addition, the developed model outperforms the conventional models for False Acceptance Rate (FAR), accuracy, and False Rejection Rate (FRR), correspondingly.
\end{abstract}

Keywords: DBN, Extraction, Face Recognition, Feature Classification, Optimization Method

\begin{tabular}{ll} 
Nomenclature & \\
\hline Abbreviations & \multicolumn{1}{c}{ Descriptions } \\
\hline PCA & Principal Component Analysis \\
LBP & Local Binary Pattern \\
FAR & False Acceptance Rate \\
DCA & Discriminant Correlation Analysis \\
FRR & False Rejection Rate \\
PMX & Partially-Mapped Crossover \\
DLSA & Discrete Lion Swarm Optimization Algorithm \\
AAM & Active Appearance Models \\
PCCycleGAN & Pose Conditional CycleGAN \\
FRR & False Rejection Rate \\
DBN & Deep Belief Network \\
\hline
\end{tabular}

\section{Introduction}

The face recognition and verification of identity progressively ensuing expediency as well as a disguise in the digital era, as well as highly exploits characteristics of biological as the verification model [1]. Nowadays research hotspot is to confirm the identification of people's rapidly, conveniently as well as efficiently as well as the research outcomes are used in numerous fields namely, identity verification in a railway station, access control, etc. Conventional verification techniques namely ID cards, access cards, work cards have progressively unsuccessful to become accustomed to the speedy pursuit of the present society, especially the railway station. Nevertheless, to cause congestion, the conventional techniques are simple, devastate a large number of peoples and material resources as well as interrupt the time of tourists [2].

In order to resolve the authentication digitalization issue the Biometrics act as an ideal solution since it exploits human physiological or behavioral characteristics in order to detect the identification of people [4]. Additionally, it has to turn out to be the major direct, user establishment as well as suitable in terms of authentication. The most complicated biometric differentiation technology is face recognition. From the viewpoint of day-to-day life applications, biometric technology is considered as the minimum directness, 
aggression as well as friendliness comparing with the existing technologies. In criminal recognition fields, e-commerce, system registration, biometric has an excellent application perspective. The additional research on face recognition from viewpoint of scientific research has significant exploration as well as hypothetical research importance in image processing, pattern recognition as well as other domains [11] and [12].

In actual fact, the extraction of feature is performed by considering the full face as 1 entity, much conventional age invariant face recognition systems might be categorized as global techniques that are contradictory for humans, to recognize a human being. The most important facial features such as geometric relationship as well as principle face modules like mouth, eyes as well as nose are generally exploited when disregarding little skin texture information.

The number of experimentations can be minimized if the classification process is simple, in the database the facial image at a coarse level is the same and entirely different regarding the investigate image. Like the feature based on to that images can be collected as gender. In a hierarchical way, facial features are grouped; the precise the face identification system is improved as well as the individual identifying information is extracted [3].

The main objective of this work is to propose a DLSA-DBN classifier for face recognition. Here, the DLSA-DBN classifier is recently adopted in that recently exploited DLSA method which trains the MLP layer in the DBN classifier.

\section{Literature Review}

In 2021, Jyothi S. Nayak and M. Indiramma [1], developed the classification of gender as preprocessing phase hence the user series examination was performed merely by the facial images. It was on the basis of the user's gender. Here, the PCA was used to generate female and male eigenspaces at the time of the training stage of the classifier. In 2020, Yanfei Liu and Junhua Chen [2], developed a PCCycleGAN for pose-invariant recognition of face and to create identity preserving and authentic frontal face images. Initially, to control particular face pose generation the conditional pose label was used. Then, to aid the identity preserving as well as synthesize realistic frontal face images was developed. In 2020, Yinghui Zhu and Yuzhen Jiang [3], exploited the LBP technique for texture features extraction of the face. In addition, extracted texture features were combined with global features extracted using 2DPCA to multifeature fusion. In 2020, Leila Boussaad and Aldjia Boucetta [4], worked on each facial module to contemplate the face as an individual module. An effectual model-based method was proposed for recognition of age-invariant exploiting a DCA. A simulation analysis was carried out in order to estimate the developed method on an extensively exploited face-aging database. In 2020, Serign Modou Bah and Fang Ming [5], presented a novel technique by exploiting the LBP model integrated with advanced image processing models namely Bilateral Filter, Image Blending Contrast Adjustment, and Histogram Equalization to recognize few problems hampering face recognition precise to enhance the LBP codes. Hence, it was exploited to enhance the precision of the whole face recognition system.

\section{Face Recognition: Proposed DLSA based DBN}

Using the classification approach, the process of face recognition is performed, which is highly beneficial as they present the class information. In this paper, the deep learning model is exploited for face recognition, and fig 1 indicates the diagrammatic representation of the proposed model. In this figure, the complete procedure takes place in 2 important schemes namely a) pre-processing b) feature extraction, and c) classification.

In the primary phase, the images, which are in the database, are subjected to the preprocessing process. From the image, to extract the facial features it is very important for classification, as well as therefore, in this paper, global, local as well as active appearance features are extracted from the image. From the k-SIFT feature, local descriptors are extracted and the newly adopted m-Co-Hog model is used to extract the global descriptors. The texture information is provided by the active appearance features, and therefore it is necessitate extracting from the face image. On the basis of conventional AAM, the active appearance features are extracted. Additionally, it is produced as a feature vector, as well as they act as input for a developed method based on DBN. For the face recognition model, this paper uses the deep learning model. On the basis of the adopted model, the optimal weights for the MLP layer of DBN are selected. For DBN classifier adopted method chooses the optimal weights. Subsequent to attaining the optimal weights from the adopted optimization technique, with the feature vector, the DBN classifiers acquire training. At last, the developed model-based DBN classifier recognizes class information for inward features. The arithmetical analysis for the adopted face recognition system is described in subsequent subsections 


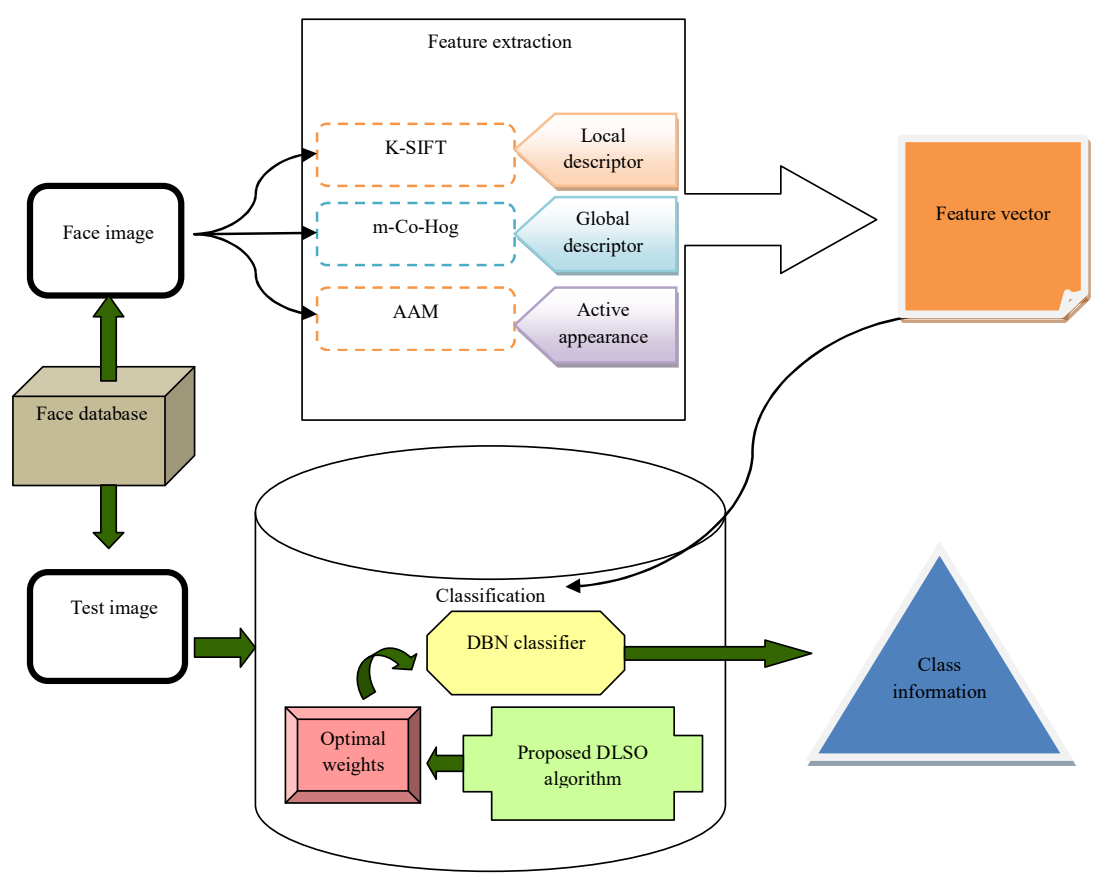

Fig. 1Architecture diagram of the proposed model

\subsection{Feature Extraction}

In face recognition, the first segment is the feature extraction from the face image. Let the $\mathrm{P}$ represents the face database, and it contains face images of $\mathrm{R}$ persons. In the database, the face images are contained which are mathematically indicated as below,

$$
\mathrm{P}=\left\{\mathrm{H}_{1}, \mathrm{H}_{2}, \ldots \mathrm{H}_{\mathrm{i}}, \ldots, \mathrm{H}_{\mathrm{R}}\right\}
$$

In eq. (1), the face image of $i^{\text {th }}$ person has indicated as $H_{i}$ in the database as well as a total number of images indicated as $\mathrm{R}$. To the feature extraction procedure, the face images are subjected, as well as the important features are extracted. In face images, features exhibit hidden texture information. Moreover, from the face image, three features are extracted, such as global descriptor, local descriptor, and active appearance features. From the k-SIFT model [7], local features are extracted, from the m-CoHog model [7] global features are extracted and using the AAM model [6] the active appearance features are extracted.

\subsubsection{Local Features Extraction using K-SIFT Model}

In this research, to extract the local features in the image, the K-SIFT technique is introduced. In the image, to detect the local space features, it is vital to place the key points within the image. To convolve the image, a Gaussian function is included, then the convolution produces the scale space for the input image $\mathrm{H}_{\mathrm{i}}$, as well as it is stated as below,

$$
\mathrm{A}(\mathrm{u}, \mathrm{v}, \delta)=\mathrm{B}(\mathrm{u}, \mathrm{v}, \delta) * \mathrm{H}_{\mathrm{i}}(\mathrm{u}, \mathrm{v})
$$

In eq. (2), the scale space is indicated as $A(u, v, \delta)$ and the Gaussian function for the transformation is indicated as $\mathrm{B}(\mathrm{u}, \mathrm{v}, \delta)$. The $(\mathrm{u}, \mathrm{v})$ states location of the input image $\mathrm{H}_{\mathrm{i}}$. In eq. (2), a scaling parameter $\delta$ is included as well as it controls scaling operation. The Gaussian function is formulated as below,

$$
\mathrm{B}(\mathrm{u}, \mathrm{v}, \delta)=\frac{1}{2 \pi \delta^{2}} \mathrm{e}^{-\frac{\left(\mathrm{u}^{2}+\mathrm{v}^{2}\right)}{2 \delta^{2}}}
$$

To determine the k-SIFT features the most important fact is to identify the DoG. For input image, the DoG is attained by the multiplication of weight constant with Gaussian function as well as it is stated as below,

$$
\begin{aligned}
& \mathrm{C}(\mathrm{u}, \mathrm{v}, \delta)=\{\mathrm{B}(\mathrm{u}, \mathrm{v}, \mathrm{w} \delta)-\mathrm{B}(\mathrm{u}, \mathrm{v}, \delta)\}^{*} \mathrm{H}_{\mathrm{i}}(\mathrm{u}, \mathrm{v}) \\
& \mathrm{C}(\mathrm{u}, \mathrm{v}, \delta)=\mathrm{A}(\mathrm{u}, \mathrm{v}, \mathrm{w} \delta)-\mathrm{A}(\mathrm{u}, \mathrm{v}, \delta)
\end{aligned}
$$


In the DoG, the determination and evade of the minimum contrast key points are considered as the subsequent step. In order to locate the minimum contrast key points, the interpolation is performed and it is represented as below,

$$
\mathrm{C}(\mathrm{r})=\mathrm{C}+\frac{\partial \mathrm{C}^{\mathrm{T}}}{\partial \mathrm{r}} \mathrm{r}+\frac{1}{2} \mathrm{r}^{\mathrm{T}} \frac{\partial^{2} \mathrm{C}}{\partial \mathrm{r}^{2}} \cdot \mathrm{r}
$$

whereas, the interpolation $r$ indicates offset point.

In order to make the interpolation task to simple, the offset point is used, and the extremum position is determined as below,

$$
\hat{\mathrm{r}}=\frac{\partial^{2} \mathrm{C}^{-1}}{\partial \mathrm{r}^{2}} \cdot \frac{\partial \mathrm{C}}{\partial \mathrm{r}}
$$

The position recognition of the unstable, the determination of the extremum point is used, and the minimum contrast key points in the image.

The determination of minimum contrast key points are formulated as below,

$$
\mathrm{w}(\hat{\mathrm{r}})=\mathrm{C}+\frac{1}{2} \frac{\partial \mathrm{C}^{\mathrm{T}}}{\partial \mathrm{r}} \cdot \hat{\mathrm{r}}
$$

Using the Hessian matrix, the determination of unstable keypoints, the principle curvature is indicated and is stated as,

$$
\mathrm{E}=\left[\begin{array}{ll}
\mathrm{C}_{\mathrm{uu}} & \mathrm{C}_{\mathrm{uv}} \\
\mathrm{C}_{\mathrm{vu}} & \mathrm{C}_{\mathrm{vv}}
\end{array}\right]
$$

The scale and position is needed to determine the feature by determining the local key points, Hence, the key points are allocated using the magnitude as well as the orientation, and it is stated as below,

$$
\begin{gathered}
\mathrm{m}(\mathrm{u}, \mathrm{v})=\sqrt{\left.(\mathrm{A}(\mathrm{u}+1, \mathrm{v})-\mathrm{A}(\mathrm{u}-1, \mathrm{v}))^{2}+\mathrm{A}(\mathrm{u}, \mathrm{v}+1)-\mathrm{A}(\mathrm{u}, \mathrm{v}-1)\right)^{2}} \\
\theta(\mathrm{u}, \mathrm{v})=\tan ^{-1}\left[\frac{\mathrm{A}(\mathrm{u}+1, \mathrm{v})-\mathrm{A}(\mathrm{u}-1, \mathrm{v})}{\mathrm{A}(\mathrm{u}, \mathrm{v}+1)-\mathrm{A}(\mathrm{u}, \mathrm{v}-1)}\right]
\end{gathered}
$$

The value of orientation and the magnitudes are assigned to create the SIFT features to present the minimum performance. To compute the local keypoints the Gaussian function is exploited, the complete performance might be corrupted. The attained SIFT features are multiplied with kernel function in order to overcome the problem, and hence, the last K-SIFT features are stated as below,

$$
\mathrm{F}_{\mathrm{k} \_ \text {SIFT }}=\mathrm{m}(\mathrm{u}, \mathrm{v}) * \mathrm{k}(\mathrm{u}, \mathrm{v})
$$

In eq. (12), $\mathrm{k}(\mathrm{u}, \mathrm{v})$ indicates multiple kernel function with the location (u,v). In $4 \times 4$ array, the attained features are computed, and, possess eight orientations. The extracted K-SIFT feature size is 128 .

\subsubsection{Global Features Extraction using m-Co-HOG Model}

The $\mathrm{m}-\mathrm{Co}-\mathrm{HoG}$ is considered as the subsequent feature extraction from the image. In [7], m-Co-HoG is described, which is an enhancement over Co-HoG features as it does not include color associated features.

\subsubsection{AAM model for Active Features Extraction}

The subsequent feature extraction is the AAM feature [6], from the image $\mathrm{H}_{\mathrm{i}}$. The active features are identified by the AAM features by image modeling. Here, from the image both texture as well as shape features are extracted using AAM.

\subsubsection{Feature Vector Construction}

From the image, subsequent to the extraction of the valuable features they are produced as the feature vector. From the $\mathrm{k}$-SIFT model, local features are extracted, and it is indicated as $\mathrm{F}_{\mathrm{k}-\mathrm{SIFT}}$. Using the mCo-Hog, the global descriptors present in the images are extracted, which is indicated as, $\mathrm{F}_{\mathrm{m}-\mathrm{Co}-\mathrm{Hog}}$. Using the AAM model, from the image, the active appearance features are extracted and it is indicated as $\mathrm{F}_{\mathrm{AAM}}$. From the image, these 3 features are extracted and they created a vector size N. Hence, for the image $\mathrm{G}_{\mathrm{i}}$ the feature vector extracted is indicated as below,

$$
\mathrm{F}_{\mathrm{i}}=\left[\begin{array}{lll}
\mathrm{F}_{\mathrm{k}-\mathrm{SIFT}} \quad \mathrm{F}_{\mathrm{m}-\mathrm{Co}-\mathrm{Hog}} \quad \mathrm{F}_{\mathrm{AAM}}
\end{array}\right]_{\times \mathrm{N}}
$$

In eq. (13), $\mathrm{F}_{\mathrm{i}}$ indicates the features extracted from the image $\mathrm{H}_{\mathrm{i}}$. As database possesses a total of $R$ images, feature attained from the complete database possess the size of $1 \times N \times R$. 


\section{DBN model}

In the face recognition procedure, the subsequent step is to build the classifier for face recognition. The deep learner is modeled by the classifier; from face image, the feature extraction needs deep learning. In this research, to resolve the face recognition model, the DBN [8] is exploited. In this paper, the DBN classifier is exploited to the adopted optimization method for the training.

For the face recognition model, in this paper, the DBN is used, which is a generally exploited model for classification. In the MLP and RBM layers, the DBN contains the input and hidden neurons. Here, a single MLP layer and 2 RBMs layers are considered by the classifier. Initially, the data is subjected to the input layer of RBM 1, and therefore, processed through several layers. To the DBN classifier, the feature input is served as the training information, and hence, the input layer of the RBM 1 is stated as,

$$
\begin{aligned}
& \mathrm{I}^{1}=\left\{\mathrm{I}_{1}^{1}, \mathrm{I}_{2}^{1}, \ldots, \mathrm{I}_{\mathrm{n}}^{1}, \ldots, \mathrm{I}_{\mathrm{n}_{1}}^{1}\right\} ; 1 \leq \mathrm{n} \leq \mathrm{n}_{1} \\
& \mathrm{~A}^{1}=\left\{\mathrm{A}_{1}^{1}, \mathrm{~A}_{2}^{1}, \ldots, \mathrm{A}_{1}^{1}, \ldots, \mathrm{A}_{\mathrm{l}_{1}}^{1}\right\} ; 1 \leq \mathrm{l} \leq \mathrm{l}_{1}
\end{aligned}
$$

In eq. (15), $\mathrm{I}_{\mathrm{n}}^{1}$ indicates $\mathrm{n}^{\text {th }}$ neuron in RBM1 layer and $\mathrm{n}$ value extends among $1 \leq \mathrm{n} \leq \mathrm{n}_{1}$. $\mathrm{A}_{1}^{1}$ indicates the $1^{\text {th }}$ hidden neuron in the RBM1 layer so that $1 \leq 1 \leq l_{1}$. Additionally, the RBM layer comprises the biases rather than features. For the RBM layer, bias available in both hidden as well as input units are indicated as below,

$$
\begin{aligned}
& \mathrm{d}^{1}=\left\{\mathrm{d}_{1}^{1}, \mathrm{~d}_{2}^{1}, \ldots, \mathrm{d}_{\mathrm{n}}^{1}, \ldots, \mathrm{d}_{\mathrm{n}_{1}}^{1}\right\} \\
& \mathrm{h}^{1}=\left\{\mathrm{h}_{1}^{1}, \mathrm{~h}_{2}^{1}, \ldots, \mathrm{h}_{1}^{1}, \ldots, \mathrm{h}_{\mathrm{l}_{1}}^{1}\right\}
\end{aligned}
$$

Here, $d_{n}^{1}$ and $h_{1}^{1}$ indicates biases available in input as well as a hidden layer of RBM1. The weights available among input as well as hidden neuron can be indicated as,

$$
\mathrm{W}^{1}=\left\{\mathrm{W}_{\mathrm{nl}}^{1}\right\} ; 1 \leq \mathrm{n} \leq \mathrm{n}_{1} ; 1 \leq \mathrm{l} \leq \mathrm{l}_{1}
$$

In eq. (18), $\mathrm{W}_{\mathrm{nl}}^{1}$ indicates the weight among the $\mathrm{l}^{\text {th }}$ hidden neuron and $\mathrm{n}^{\text {th }}$ input neuron. On the basis of the bias and the weight, the output of the RBM 1 is calculated which is available in input as well as hidden neurons, and therefore, RBM1 output is formulated as below,

$$
\mathrm{X}_{\mathrm{l}}^{1}=\sigma\left[\mathrm{h}_{\mathrm{l}}^{1}+\sum_{\mathrm{n}} \mathrm{I}_{\mathrm{n}}^{1} \mathrm{~W}_{\mathrm{nl}}^{1}\right]
$$

In eq. (19), for the output calculation $\sigma$ indicates the activation function. The output neuron available in RBM 1 is calculated as,

$$
\mathrm{X}^{1}=\left\{\mathrm{X}_{1}^{1}\right\} ; 1 \leq 1 \leq \mathrm{l}_{1}
$$

Likewise, the neurons and the layers belong to RBM2 are initialized as well as therefore, output calculated by RBM 2 is indicated as $\mathrm{X}^{2}$. To the MLP layer, RBM2 output is subjected as a sample. Here, the MLP layer possesses input, hidden as well as weight neurons beside their equivalent weights as well as bias. The input neurons comprising MLP layers are stated as below,

$$
\mathrm{z}=\left\{\mathrm{z}_{1}, \mathrm{z}_{2}, \ldots, \mathrm{z}_{1}, \ldots, \mathrm{z}_{1}\right\}=\left\{\mathrm{h}_{1}^{2}\right\} ; 1 \leq \mathrm{l} \leq \mathrm{l}_{1}
$$

Since the RBM 2 output is subjected to the MLP layer, it possesses a similar layer as the RBM 2 . The hidden units of MLP are stated as below,

$$
\mathrm{p}=\left\{\mathrm{p}_{1}, \mathrm{p}_{2}, \ldots, \mathrm{p}_{\mathrm{q}}, \ldots, \mathrm{p}_{\mathrm{q}_{1}}\right\} ; 1 \leq \mathrm{q} \leq \mathrm{q}_{1}
$$

In eq. (22), $\mathrm{p}_{\mathrm{q}}$ indicates the $\mathrm{q}^{\text {th }}$ hidden neuron of MLP. The MLP output presents classified information, is stated as,

$$
\mathrm{O}=\left\{\mathrm{O}_{1}, \mathrm{O}_{2}, \ldots, \mathrm{O}_{\eta}, \ldots, \mathrm{O}_{\eta_{1}}\right\} ; 1 \leq \eta \leq \eta_{1}
$$

In eq. (23), $\eta_{1}$ states the total output neurons in the MLP. In both inputs as well as a hidden layer, MLP possess the weights and they are stated as below,

$$
\mathrm{W}^{\mathrm{I}}=\left\{\mathrm{W}_{\mathrm{lq}}^{\mathrm{I}}\right\} ; 1 \leq \mathrm{l} \leq \mathrm{l}_{1} ; 1 \leq \mathrm{q} \leq \mathrm{q}_{1}
$$

In eq. (24), $\mathrm{W}_{\mathrm{lq}}^{\mathrm{I}}$ indicates the weight among $\mathrm{l}^{\text {th }}$ input neurons as well as $\mathrm{q}^{\text {th }}$ hidden neurons. In MLP, The output presented by the hidden layer is stated as, 


$$
\mathrm{p}_{\mathrm{q}}=\left[\sum_{\mathrm{l}=1}^{\mathrm{l}_{1}} \mathrm{w}_{\mathrm{lq}}^{\mathrm{I}} * \mathrm{j}_{\mathrm{l}}\right] \beta_{\mathrm{q}} \forall \mathrm{j}_{\mathrm{l}}=\mathrm{h}_{\mathrm{l}}^{2}
$$

In eq. (26), for the MLP, $\beta_{\mathrm{q}}$ indicates the bias presented in the input neuron. The weight available among the hidden and the output layer is stated as,

$$
\mathrm{W}^{\mathrm{H}}=\left\{\mathrm{W}_{\mathrm{q \eta}}^{\mathrm{H}}\right\} ; 1 \leq \mathrm{q} \leq \mathrm{q}_{1} ; 1 \leq \eta \leq \eta_{1}
$$

For the MLP the ultimate output is stated as below,

$$
\mathrm{O}_{\eta}=\sum_{\eta=1}^{\eta_{1}} \mathrm{~W}_{\mathrm{q \eta}}^{\mathrm{H}} * \mathrm{p}_{\mathrm{q}}
$$

In eq. (27), $\mathrm{W}_{\mathrm{q \eta}}^{\mathrm{H}}$ indicates the weight among the hidden neuron and output of MLP.

\section{Proposed DLSO}

(i) Discrete Coding

From the conventional real value coding, the lion population alters to integer discrete coding. Here, the individual lion is indicated as an array length $\mathrm{n}$, as well as every element value in the array is represented as a positive integer among 1 as well as $n$, indicating equivalent layer, to fulfill the Hamilton loop condition, there are no elements with a similar value in array [10].

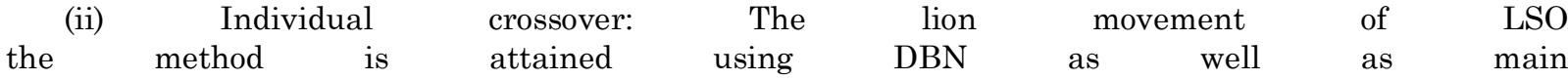
equations are to adjust the numerical value of elements in

individual.
In the Genetic Algorithm,, the crossover operator is developed can enable offspring to retain structural information of parent, creating individuals movement highly efficient. In the Genetic Algorithm, conventional crossover operators comprise PMX, cycle crossover (CX), shuffle crossover, order crossover (OY), and uniform crossover [10].

On basis of this experimentation, the OY operator will obtain a superior solution in the Genetic Algorithm; the OY operator is exploited in DLSO. It produces the offspring by selecting a sub-tour from 1 parent as well as conserving the relative order of layers of the other parent. The OY works are stated as below:

Arbitrarily choose two cut points mark stated with "|" on parents

$\mathrm{PAR}_{1} \mathrm{AND} \mathrm{PAR}_{2}$

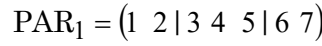

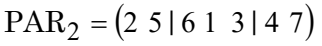

In the following manner produce offspring: initially, the sub-tour among the two cut points are copied into offsprings:

$$
\begin{gathered}
\mathrm{O}_{1}=\left(\begin{array}{lll}
--\mid 3 & 4 & 5
\end{array} \mid--\right) \\
\mathrm{O}_{2}=\left(\begin{array}{lll}
--\mid 6 & 1 & 3
\end{array} \mid--\right)
\end{gathered}
$$

Subsequently, initiating from the second cut point of a parent, in similar order the
bits from the conventional bits are canceled. Take producing $\mathrm{O}_{1}$ as an instance. The series of bits in PAR from the second point already is $\quad(4$ 4 Initiating from the second $\mathrm{O}_{1}$, obtained

$$
\begin{aligned}
& \mathrm{O}_{1}=\left(\begin{array}{ll|llll}
6 & 1 \mid 3 & 4 & 5 & 7 & 2
\end{array}\right) .
\end{aligned}
$$

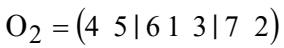

Thee lion king movements, lioness and cubs are finished on the basis of the following formulations

$$
\begin{aligned}
& y_{i}^{t+1}=p_{i}^{t} \text { OY g } \\
& y_{i}^{t+1}=p_{i}^{t} O Y p_{c}^{t}
\end{aligned}
$$




$$
\mathrm{y}_{\mathrm{i}}^{\mathrm{t}+1}=\left\{\begin{array}{l}
\mathrm{p}_{\mathrm{i}}^{\mathrm{t}} \text { OY } \mathrm{g}^{\mathrm{t}}, \mathrm{q} \leq \frac{1}{3} \\
\mathrm{p}_{\mathrm{i}}^{\mathrm{t}} \text { OY } \mathrm{p}_{\mathrm{m}}^{\mathrm{t}}, \frac{1}{3}<\mathrm{q} \leq \frac{2}{3} \\
\mathrm{p}_{\mathrm{i}}^{\mathrm{t}} \mathrm{OY} \mathrm{g}^{-\mathrm{t}}, \frac{2}{3}<\mathrm{q} \leq 1
\end{array}\right.
$$

$\mathrm{p}_{\mathrm{i}}^{\mathrm{t}}, \mathrm{g}^{\mathrm{t}}, \mathrm{p}_{\mathrm{c}}^{\mathrm{t}}, \mathrm{p}_{\mathrm{m}}^{\mathrm{t}}, \mathrm{g}^{-\mathrm{t}}$ indicates the optimal historic position of a lion, the optimal historic position of $\boldsymbol{t}^{\boldsymbol{t h}}$ generation, the best historical location of a lioness arbitrarily chosen from the $\boldsymbol{t}^{\boldsymbol{t} \boldsymbol{h}}$ generation lioness population.; OY $\quad$ states the OY operation.

(iii) Initiation of $\mathrm{C} 2$-opt operator: By separating the population into 3 classifications-lioness, lion king, and lion cub The lion swarm the method offers a strong global optimization capability. The particular approach is that in each iteration, $\mathrm{C} 2$ - opt operation is carried out on lion king, two optimal lionesses, as well as the best lion cub.

\section{Result And Discussion}

The complete face recognition experimentation with the adopted DLSO- based DBN classifier has experimented with in the MATLAB tool. The adopted model-based DBN classifier was experimented by taking into consideration the images from the CVL database [9].

The 114 persons' face images have available in the database. The experimentation measures state that the performance of classification, and in this paper, the adopted based DBN classifier performance was performed on the basis of the measures, namely accuracy, FRR, and FAR.

Table 1 summarizes the comparative analysis of several conventional models exploited in this paper, as well as these performances, are evaluated with the adopted DLSO-DBN classifier. The performance analysis is performed over measures, namely FAR, accuracy, and FRR, correspondingly. Here, the proposed method is compared with the SIFT, Histogram of Oriented Gradients (HOG), and WHOG. Moreover, the proposed method is $20 \%$ better than the SIFT, $16 \%$ better than the SIFT, and $18 \%$ better than the SIFT for accuracy. Then the the proposed method is $15 \%$ better than the SIFT, $18 \%$ better than the SIFT, and 19\% better than the SIFT for FRR. Subsequently, the proposed method is $19 \%$ better than the SIFT, 16\% better than the SIFT, and 22\% better than the SIFT for accuracy The adopted classifier outperforms the conventional models with maximum accuracy value. Additionally, the FAR and the FRR of the adopted model are the least while comparing with the conventional models.

\section{Table 1 Performance analysis of the proposed model}

\begin{tabular}{|c|c|c|c|}
\hline \multirow[t]{2}{*}{ Methods } & \multicolumn{3}{|c|}{ Evaluation metrics } \\
\hline & Accuracy & FRR & FAR \\
\hline SIFT & 0.9773 & 0.0191 & 0.0191 \\
\hline WHOG & 0.9717 & 0.0173 & 0.0173 \\
\hline HOG & 0.3974 & 0.1455 & 0.1455 \\
\hline k-SIFT & 0.9579 & 0.01 & 0.01 \\
\hline Proposed classifier & 0.99 & 0.0077 & 0.0077 \\
\hline
\end{tabular}

\section{Conclusion}

This work proposes a face recognition method, which was exploited by a deep learner. For the classification, the DBN was used by the developed classifier and few renowned features namely, AAM, kSIFT, as well as the newly adopted m-Co-Hog features, which were extracted from the image for the DBN training. In the image, the features define the global, local as well as active appearance descriptors. Here, the information concerning the face image was provided by the features and hence aid in face recognition. From the face image, the features were extracted and form as a vector, and it was presented to DBN as training input. Using the recently developed DLSA method the DBN weights were optimally selected in the training phase. Subsequent to training, the classified information was provided by the DBN classifier for the test face image. 


\section{References}

[1] Jyothi S. NayakM. Indiramma,"An approach to enhance age invariant face recognition performance based on gender classification",Journal of King Saud University - Computer and Information SciencesAvailable online, 14 January 2021.

[2] Yanfei LiuJunhua Chen,"Unsupervised face Frontalization for pose-invariant face recognition", Image and Vision Computing,13 December 2020.

[3] Yinghui ZhuYuzhen Jiang,"Optimization of face recognition algorithm based on deep learning multi feature fusion driven by big data",Image and Vision Computing,18 September 2020.

[4] Leila BoussaadAldjia Boucetta,"An effective component-based age-invariant face recognition using Discriminant Correlation Analysis",Journal of King Saud University - Computer and Information SciencesAvailable online, 25 August 2020

[5] Serign Modou BahFang Ming,"An improved face recognition algorithm and its application in attendance management system",Array, 26 December 2019.

[6] A.U. Batur, M.H. Hayes, "Adaptive active appearance models", IEEE Transactions on Image Processing, vol. 14, no. 11, pp. 1707 - 1721, Nov. 2005.

[7] Hima Bindu, Manjunathachari K., "Hybrid feature descriptor and probabilistic neuro-fuzzy system for face recognition", Sensor Review, vol. 38, no. 3, pp.269-281, 2018.

[8] Bc. Ján Vojtech, "Deep Neural Networks and Their Implementation", Thesis, Charles University in Prague, 2016.

[9] CVL database, taken from "http://www.lrv.fri.uni-lj.si/facedb.html", accessed on May 2018.

[10] B. R. Rajakumar,"The Lion's Algorithm: A New Nature-Inspired Search Algorithm", Procedia Technology2012.

[11] K.Srinivas,"Prediction of E-Learning Efficiency by Deep Learning in EKhool Online Portal Networks", Multimedia Research, vol 3, no. 4, October 2020.

[12] Arvind Madhukar Jagtap,"Developing Deep Neural Network for Learner Performance Prediction in EKhool Online Learning Platform", Multimedia Research, vol. 3, no. 4, October 2020. 